\title{
¿Primero los nuestros? Apertura controlada de fronteras y cosmopolitismo moderado
}

\author{
Our people first? Controlled border \\ opening and moderate cosmopolitanism
}

Federico Arcos Ramírez

Universidad de Almería farcos@ual.es

Orcid: 0000-0002-3578-3637

DOI: http://dx.doi.org/10.15366/bp.2020.23.003

Bajo Palabra. II Época. No23. Pgs: 73-100 
Este trabajo ha sido elaborado en el marco de los proyectos de investigación UAL-FEDER

"Control de fronteras y Derechos Humanos en el Mediterráneo" (UAL18-SEJ-C004-B) y

"Fronteras, democracia y justicia global" (PGC2018-093656-B-100).

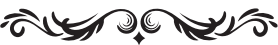

\section{Resumen}

Un argumento recurrente en las tesis de quienes abogan por el mantenimiento del actual modelo de fronteras cerradas es el que se conoce como favoritismo o prioridad de los conciudadanos. Según este argumento, los Estados tendrían derecho a controlar y limitar la entrada de extranjeros en su territorio porque, de lo contrario, no podrían mantener un sistema socioeconómico que requiere una atención especial a las necesidades de sus miembros menos favorecidos. Este artículo desarrolla una crítica de ese argumento sin negar el conflicto práctico entre las lealtades patrióticas y las responsabilidades distributivas globales analizando si éstas son lo suficientemente fuertes como para soportar el peso del valor intrínseco de todos los seres humanos y sus necesidades más básicas y urgentes.

Palabras clave: fronteras, conciudadanos, prioridad de los compatriotas, justicia global, derechos humanos, necesidades básicas, fronteras abiertas.

\section{Abstract}

A recurrent argument in the theses of those who advocate for maintaining the current model of closed borders is the one known as favoritism or priority of fellow citizens. According to this argument, States would be entitled to control and limit the entry of foreigners into its territory because, otherwise, could not maintain a socioeconomic system that requires special attention to the needs of its less advantaged members. This article develops a critique of this argument without denying the practical conflict between the patriotic loyalties and global distributive responsibilities analyzing whether these are strong enough to withstand the weight of the intrinsic value of all human beings and their most basic and urgent needs.

Keywords: borders, fellow citizens, priority of compatriots, global justice, human rights, basic needs, open borders. 


\section{El conflicto entre la justicia doméstica y las responsabilidades globales}

TANTO LOS GOBIERNOS como muchas veces los ciudadanos del primer mundo consideran que las responsabilidades especiales que tienen con los compatriotas constituyen una razón ética poderosa para resistirse al cumplimiento de sus obligaciones de justicia global; entre otras, acabar con el hambre y la pobreza mundial, apostar por un régimen del comercio internacional menos proteccionista para sus productos, condonar la deuda externa, etc. En particular, estiman que hay una tensión importante entre garantizar las prestaciones Welfare State y dedicar más fondos a la ayuda al desarrollo y abrir las fronteras políticas a la inmigración. ¿¿Significa todo ello que existe un conflicto entre los deberes de justicia hacia los conciudadanos y las responsabilidades globales? En tal caso, ¿estaría justificado otorgar prioridad a las necesidades de nuestros conciudadanos frente a las de los demás seres humanos?

Para algunos, dicha preferencia no deja de ser una forma -si se quiere sofisticada- de egoísmo, una coartada para perpetuar la posición de ventaja de la que ya disfrutan algunos en el acceso a los recursos y la riqueza del mundo. Es más, estiman que los vínculos patrióticos no generan auténticos deberes morales. En primer lugar, porque, como señalan todas las tradiciones ético-filosóficas importantes, éstos únicamente pueden originarse adoptando un punto de vista imparcial o impersonal, independiente de la identidad o los intereses propios o de las personas que con las que uno tiene vínculos especiales, lo que, en la práctica, exige tratarlas por igual. Y estas son, precisamente, el tipo de razones que han jugado el peso más significativo en la justificación de las exigencias de la justicia global que los Estados, como vemos, se resisten a cumplir. En segundo lugar, porque se trata de un vínculo dependiente del azar y, como tal, éticamente irrelevante. Como sostienen algunos cosmopolitas, la función de la justicia es, precisamente, mitigar y compensar los efectos que ejerce sobre las opciones de vida de millones de seres humanos el poseer una $\mathrm{u}$ otra nacionalidad no elegida.

Otros pensadores estiman, por el contrario, que, si la resistencia de los países más ricos obedeciera únicamente al puro autointerés, la misma no conllevaría ningún rompecabezas moral o filosófico. Detrás de la práctica de los gobiernos y los individuos existen creencias e intuiciones muy arraigadas que consideran moralmente correcto otorgar preferencia en ciertos casos a los intereses y necesidades de aquellos individuos con los que estamos relacionados de un modo especial (Caney 2005: 
134). Por consiguiente, es posible que, tras la resistencia de los países más prósperos a cumplir con sus deberes globales, no encontremos un comportamiento egoísta sino, más bien, un modo (aceptable o no) de resolver la tensión entre dos tipos de exigencias éticas diferentes. Para Seglow (2010), estaríamos ante un conflicto entre la "moralidad del sentido" común y la perspectiva cosmopolita, mientras que Abizadeh y Gilabert (2008) estiman que ambos tipos de razones formarían parte de la "moralidad del sentido común".

Para sostener esta posición recurren básicamente a dos tipos de argumentos. El primero considera que, en el plano fundacional, existirían dos tipos de razones éticas. Por un lado, las basadas en la imparcialidad universal, que exigen tratar a todos los seres humanos de la misma manera y que serían el fundamento de deberes generales. Por otro, las que derivan directamente de las relaciones que tenemos con ciertas personas y que fundamentan deberes y responsabilidades morales especiales. De estas últimas se afirma que brotan directamente de la relación, que son constitutivas de ésta (Miller 2007: 35; Miller 2016: 26), que surgen de hechos (el ser hermano, colega o compatriota) que representan un "momento ético independiente de cualesquiera otros rasgos más fundamentales de dichas relaciones" (Wellman 2000: 539). Son, como dirá Scheffler, razones o deberes "underived". Esta postura encierra una contradicción, que da pie al conocido como "dilema de Nussbaum":

O sostenemos que dedicar una atención especial a ciertas personas es un medio efectivo de hacer el bien a la humanidad en su conjunto, o debemos suponer que esas personas simplemente tienen más valor que las otras. Lo que no podemos hacer es afirmar que todas las personas tienen el mismo valor $y$, al mismo tiempo, insistir en que nuestras relaciones hacia ciertas personas nos obligan a dedicar especial atención a las mismas con independencia de que, al hacerlo, promovamos o no el bien del conjunto de la humanidad (Scheffler 2001: 118).

Conscientes de las dificultades que entraña hacer compatibles en el plano fundacional las responsabilidades especiales y el principio (cosmopolita) de la igualdad moral de todos los individuos, otros teóricos esquivan el lenguaje de los deberes especiales asociativos y el tipo de dualismo ético que alimenta su justificación. Frente a ello, siguen una metodología centrada en demostrar que el valor de la igualdad ética de todos los seres humanos únicamente genera demandas redistributivas basadas en la igualdad comparativa en un determinado contexto, el que conforman los miembros de una comunidad política (Blake 2002: 257-258; Sangiovanni 2007: 3-39). Proponen, de este modo, una rescritura del liberalismo capaz de hacer compatible la universalidad racional de la igualdad con el alcance limitado de ciertos derechos (políticos, socioeconómicos, etc.) reconocibles únicamente a los compatriotas. 
El conflicto al que aludimos ¿es real o solo aparente? ¿Constituyen los deberes distributivos hacia los conciudadanos auténticas razones éticas independientes de las globales? En caso de responder afirmativamente ¿̇ería en algún supuesto justificable anteponer su cumplimiento a las responsabilidades hacia los extranjeros que se encuentran peor?

Es necesario distinguir entre el favoristismo y la prioridad de los compatriotas. Como veremos, los defensores de una concepción estatista de la justicia global consideran que los miembros de una comunidad política se deben entre sí deberes de justicia más exigentes que los que tienen frente al resto de seres humanos. Mientras los primeros serían exigencias basadas en la igualdad comparativa, las responsabilidades globales serían exigencias más modestas, no ya de justicia, sino de beneficencia o humanidad o, en el caso de considerarlas de justicia, de un contenido suficientista o prioritarista, pero no igualitarista. Al margen de que esta concepción resulte o no preferible a la globalista y/o genuinamente cosmopolita, para la que la justicia global debería adoptar el mismo principio distributivo que la doméstica, ¿hay una solución de continuidad entre sostener que frente a los compatriotas tenemos deberes distintos, especiales y más ambiciosos en su contenido y que, en caso de colisionar con la justicia global, los primeros gocen de prioridad frente a estos últimos? La especialidad de la esfera de los compatriotas ¿̇implica conceptualmente también su prioridad?

\section{Los presupuestos del conflicto}

Conviene aClarar que el posible conflicto entre el polo ético de los compatriotas y el del resto de los seres humanos se referirá, al menos en principio, exclusivamente a las obligaciones positivas y no a las negativas. El principal contexto en el que se vienen plateando posibles tensiones entre nuestros deberes globales y las responsabilidades hacia los compatriotas es, básicamente, el de la justicia distributiva y, sólo excepcionalmente, el de la prohibición de atentar contra la vida e integridad física de las personas que exigen los derechos civiles. Sin embargo, algunas decisiones de los gobiernos entrańan también conflictos entre mejorar el bienestar de los compatriotas y no menoscabar los deberes negativos globales, entre otros, a través del comercio de material militar a países que no respetan significativamente los derechos humanos ${ }^{1}$.

\footnotetext{
${ }^{1}$ Los acuerdos suscritos por el grupo público Navantia para construir cinco corbetas para la Armada saudí serían un buen exponente de dicho conflicto. Los partidos políticos que defienden dicho acuerdo esgrimen en su favor la creación de 6.000 puestos de trabajo durante los próximos cinco años en los astilleros de Puerto Real. Las
} 
En el plano de las responsabilidades distributivas, la tensión entre ambos polos descansaría en los tres siguientes presupuestos:

1) Es posible determinar el contenido de los principios y deberes de la justicia (distributiva) doméstica o estatal antes de preguntarse por los de la global (Seleme 2007: 101-102).

2) Existen situaciones en las que no hay recursos suficientes para hacer compatible el cumplimiento simultáneo y pleno de ambas exigencias. No se trataría de una escasez severa de recursos, sino de una más moderada, que obliga ponderar unas y otras exigencias. Un escenario del primer tipo parecería situarnos fuera de las circunstancias de la justicia social (Tan 2013: 42-43).

3) No sólo los deberes distributivos globales, sino también los que tienen como destinatarios a los conciudadanos, constituyen razones morales independientes.

4) Resulta inevitable priorizar, en mayor o menor grado, la observancia de un tipo de obligaciones sobre las otras.

La solución al conflicto puede proceder de las dos siguientes maneras:

1) Negando que estemos ante un conflicto real y no aparente ya que:

a. Los principios de la justicia doméstica y los de la justicia global no pueden, al menos en cierta medida, ser decididos separadamente.

b. No existe una escasez moderada de recursos que impida, en el plano empírico, la satisfacción plena, conjunta y simultanea de ambas exigencias. Ello dependerá no sólo de la cuantía de tales recursos sino también de cuál sea el tipo de principio distributivo en el que se funden tanto los deberes de la justicia doméstica como, y esto resulta menos pacífico, los de la global.

ONG y los partidos que se oponen al acuerdo denuncian que dichas embarcaciones van a ser empleadas en un conflicto armado, como es la guerra civil en Yemen, en el que se podrían utilizarse para cometer crímenes de lesa humanidad e infracciones graves de los Convenios de Ginebra u otros crímenes de guerra. Cabría hablar, pues, de un conflicto entre beneficiar a los menos aventajados de la misma comunidad política y no dańar (de forma indirecta) a otros seres humanos. 
c. Los vínculos especiales, en particular los patrióticos, no constituyen auténticas razones morales independientes de las universales que permita hablar, pues, de una tensión real entre ambas.

2) Aceptando que el conflicto es real y otorgando una preferencia, en función de los casos, a un tipo de razones sobre otras.

\section{Entre la teoría ideal y no ideal}

Comenzaré el Desarrollo de una PROpUesta acerca de cómo podría no tanto resolverse como, más modestamente, dibujarse mejor el conflicto referido sin cuestionar que los principios de la justicia doméstica y la justicia global pueden ser decididos separadamente. A mi juicio, cualquier intento de justificar tanto el favoritismo como, sobre todo, la prioridad de los compatriotas no puede cuestionar esta premisa. Y, lo que, es tanto o más destacable aún, prima facie, no puede desarrollarse en el marco de una teoría de la justicia no ideal (Couture y Nielsen 2006: 190).

Para una teoría puramente ideal, esta solución no resultaría aceptable. El hecho de que un cierto grupo de personas pertenezca a la misma comunidad política sería una circunstancia éticamente irrelevante a la hora de otorgar prioridad a las necesidades de unos individuos frente a las de otros (Beitz 1999: 209; Ypi 2013: 77-78). No parece que en el plano ideal haya que tener en cuenta más datos que la igualdad moral de todos los seres humanos y de sus necesidades e intereses básicos.

Prima facie, en un plano ideal y abstracto, la igualdad moral de todos los seres humanos no resultaría únicamente incompatible con otorgar ningún tipo de prioridad a los compatriotas, con sacrificar, en mayor o menor medida, la justicia global en beneficio de la doméstica, sino también con la posibilidad de mejorar la posición de los conciudadanos, si ello no supone un perjuicio para el resto de las personas. Para una teoría ideal esta opción tampoco tendría cabida ya que, como justicia de máximos, perfecta y bajo condiciones favorables (recursos, instituciones, motivación), habría de adoptar un criterio distributivo estrictamente igualitario en sentido comparativo. Por tanto, en dicho plano no sólo solo no cabe admitir deberes de prioridad que justifiquen anteponer las necesidades o intereses de los compatriotas frente el resto de las personas, sino tampoco deberes adicionales que permitan incrementar, reforzar o mejorar respecto a los conciudadanos los deberes positivos generales (Abizadeh 2016: 108).

Sin embargo, esta última conclusión podría no ser tan rotunda si nos situamos en un plano pragmático y admitimos que, junto a la concurrencia de circunstan- 
cias favorables (reparto equitativo y adecuado de los recursos, instituciones capaces de implementar los principios, etc.) y ciertas consideraciones fácticas mínimas relativas a ciertos rasgos generales de la condición humana (altruismo limitado, vulnerabilidad, interés en tener planes de vida, etc.), la teoría ideal también debería incluir la necesidad que tienen todas las personas en acceder a relaciones especiales y a los vínculos y dependencias que surgen entre los que participan en ellas. Bajo tales circunstancias, algunos globalistas no ven objeción el admitir ciertas formas de privilegio de los compatriotas, ya que consideran compatible con la igualdad moral que dichas relaciones permitan al agente incrementar lo que debe a los miembros de su propio grupo o comunidad, siempre y cuando no se disminuya lo que se debe a cualquier otra persona (Pogge 2002: 78-79; Tan 2004:153; Seglow 2010: 66).

En la teoría ideal, las exigencias que derivan de las relaciones entre conciudadanos sólo podrían empezar a ser tomadas en consideración una vez que se han decidido y satisfecho las responsabilidades globales (Beitz 1999: 170). Como señala Shue, la justicia no puede empezar por casa, sino que los principios de la justicia global han de ser establecidos simultáneamente, incluso con anterioridad, a los de la doméstica. Para determinar los deberes de ésta es preciso conocer con antelación los recursos y riqueza que nos corresponden legítimamente, y esto es algo que no puede ser establecido sin tener en cuenta a los demás pueblos y estados (Shue 1983: 603; Nielsen 2003: 284-285).

La tesis de Shue parece unida a la creencia en que la justicia global ideal y perfecta demanda un Estado global soberano (Sen 2009: 25), o, como mínimo, establece una presunción en favor de este último (Nili 2015: 242). En su acepción más genuina, éste conllevaría la desaparición de las actuales formas de membresía política en favor de una ciudadanía mundial. En una versión menos maximalista y flexible, permitiría ciudadanías múltiples, pero, en cualquier caso, no podría convertir a las formas de pertenencia no globales en un factor relevante a la hora de distribuir recursos escasos, ni, por tanto, aceptar la posibilidad de concebir la justicia doméstica al margen de la global.

No obstante, sólo una minoría de cosmopolitas o globalistas suscribirían esta última tesis (Wendt 2003: 491-542; Marchetti 2008; Cabrera 2010). En ellos han pesado mucho los temores expresados hace siglos por Kant, y más recientemente por Rawls y Pettit, de que un Estado mundial, entendido como un régimen político unificado con los poderes reconocidos a los gobiernos nacionales, sería un despotismo global (Rawls 1999: 49). De ahí que la mayoría de especialistas comparta la visión de que una futura democracia global no debe articularse a través de un federalismo mundial o cosmo-federalismo que incluya unos poderes legislativo, ejecutivo y judicial mundiales, sino que debe ser concebida como una gobernanza 
mundial postsoberana, multilateral, policéntrica, sustentada en una dispersión y fragmentación de la soberanía en diferentes agencias con diversos tamańos que dan lugar a un sistema de autoridades superpuestas. Un Estado mundial equipado con la parafernalia convencional de estado moderno, en particular con la monopolización del uso de la fuerza, no es ni necesaria, ni deseable (Scheuerman 2013; Bayón 2014).

Cuestionada la factibilidad y deseabilidad política de un poder superior centralizado, parece más razonable optar por una concepción de la justicia global que intente encontrar un punto de encuentro entre las exigencias de la teoría ideal genuinamente cosmopolita y algunas circunstancias del mundo en el que vivimos que, aunque no sean estructurales y necesarias, constituyen realidades que difícilmente van a desaparecer y, más aún, que no está claro ni siquiera que sea deseable que lo hagan. Por tanto, aunque un mundo integrado por Estados no sea una realidad irrebasable (Chatterjee 2011: 200-201), una teoría de la justicia global que persiga un equilibrio razonable entre lo moralmente deseable y lo políticamente factible podría aceptar que se establezcan, en primer lugar, las exigencias de la justicia doméstica y, en un segundo momento, las de la justicia global, sin perjuicio de que estas últimas puedan obligar a modificar, atemperar, corregir e, incluso en algunos casos eliminar, las primeras. La prioridad temporal en la determinación de los principios de la justicia doméstica se justificaría, pues, por el carácter estatal de la sociedad internacional, que hace mucho más realizable un cumplimiento de la justicia global como corrección y/o limitación de la justicia doméstica que como un conjunto de deberes que deben ser establecidos tomando únicamente en consideración la igualdad moral de todos los seres humanos.

De ahí también la conveniencia de optar por una idea de la justicia global, tal y como defiende Sen, menos centrada en los esquemas que en las realizaciones, más preocupada en acabar aquí y ahora con las injusticas globales (y con aquellas manifestaciones de la prioridad de lo compatriotas que injustificadamente contribuyan a crearlas o exacerbarlas) que en tratar de llevar a la práctica los principios de la teoría perfecta (Sen 2009: 5; Shklar 2018). Por tal razón, la solución a los problemas que demandan una justicia global no puede ignorar el papel que los actuales Estados podrían y deberían desempeñar de cara a lograr las trasformaciones que conduzcan a un mundo mejor. Lo contrario representaría "una oportunidad perdida para promover objetivos claves de la justicia global” (Brock 2015: 113). La operatividad de medidas de justicia distributiva global como la famosa tasa Tobin, la menos conocida "Bin Tax", el Global Fund Tax (Steiner), la Cuota Global (Casal) o la condonación de la deuda externa, no está supeditada a la desaparición de los Estados y/o los esquemas de justicia doméstica, sino que deben hacerse posibles a partir de ellos. 


\section{Los deberes especiales hacia los compatriotas como razones éticas independientes}

Muchos cosmopolitas Rechazan que en el plano fundacional exista la dualidad de razones éticas (universales y particulares) antes señalada como uno de los presupuestos del posible conflicto entre las responsabilidades distributivas locales y domésticas. Una de las expresiones más conocida de este escepticismo es la tesis de Goodin según la cual las lealtades hacia ciertas personas o grupos serían únicamente deberes generales distribuidos, instrumentos por medio de los cuales la comunidad moral consigue asignar deberes generales a agentes particulares (Goodin 1998: 678).

Un cosmopolitismo más moderado ha defendido que, aunque sea correcta la tesis de Scheffler de que tales relaciones son "underived", y que su valor no depende de que sean un medio eficaz para realizar el mejor estado de cosas, ello no permite concluir, sin más, que posean también un valor ético incondicional. Las relaciones especiales poseen valor moral, y no sólo prudencial, cuando no violan ciertas constricciones, entre otras, el idéntico valor del bienestar de todos y cada uno de los seres humanos. Por sí solas, al margen de que respeten esta condicionalidad, las relaciones especiales no proporcionan razones básicas, suficientes o últimas para reconocer responsabilidades morales especiales. A la vista de ello, el conflicto entre las responsabilidades especiales (incluidas las que tienen como destinatarios a los compatriotas) y los deberes generales hacia todos los seres humanos, basados en la igualdad moral de todos ellos, se desarrollaría dentro de este último valor, más concretamente, en el seno de la igualdad en el acceso a ciertos bienes básicos para el bienestar humano (a ciertos recursos y a esas mismas relaciones especiales). Admitido esto, los autores señalados señalan no sólo que la igualdad moral cosmopolita in abstracto condiciona el valor ético de las relaciones especiales, sino también que los deberes generales pueden operar como esa condición y, por tanto, evitar el surgimiento de deberes especiales (Abizadeh y Gilabert 2008: 360-362).

Aunque comparto tanto su defensa del valor no instrumental de las relaciones especiales, como la existencia de límites o condiciones para atribuir un valor moral definitivo a éstas, no coincido con Abizadeh y Gilabert en su modo de entender las nociones de conflicto entre deberes morales, razones éticas prima facie y, a la postre, de deberes éticos genuinos. A su juicio, no existirían auténticos conflictos entre requerimientos morales como, por ejemplo, los derivados de las relaciones especiales y los deberes generales, ya que los primeros sólo serían deberes de forma aparente, a primera vista, ya que la noción de deber sólo se aplica a lo que debe hacerse, consideradas todas las circunstancias. Es decir, antes de establecer lo que debe concluyentemente hacerse, no habría genuinos deberes. Frente este planteamiento 
kantiano, en lugar de afirmar que las responsabilidades especiales únicamente poseen valor moral una vez constatado que no violan o traspasan los mencionados valores últimos cosmopolitas y de negar, pues, que entre las primeras y los deberes generales pueden surgir auténticas tensiones, considero más razonable sostener que aquellas poseen un valor moral pro tanto (Kagan 1991: 17). Como tales, cuando colisionan con los deberes generales y/o los valores cosmopolitas, pueden terminar resultando mermadas o socavadas por estos últimos, pero no dejan, por ello, de ser razones morales, ni quedan anuladas por tales valores (Lenard y Moore 2009: 400). Defenderé, pues, que los vínculos con los conciudadanos poseen valor ético, no en el sentido de que generen deberes que establezcan concluyentemente lo que debe hacerse, sino en el de constituir razones a las que cabe reconocer cierto peso moral, el suficiente para que puedan y deban ser tomadas en consideración en una deliberación dirigida a determinar las responsabilidades distributivas de los Estados.

\section{La relevancia ética de la cooperación entre conciudadanos}

Hemos ADMitido QUe los vínculos con los compatriotas constituyen razones éticas independientes (aunque sea solo pro tanto) de las razones generales. Sin embargo, no hemos señalado qué criterio distributivo adoptarían y sobre la base de qué razones o fundamentos. Antes de hacerlo, conviene distinguir entre la ética de la obtención o reconocimiento de la ciudadanía y el valor ético de está, esto es, entre la justicia de los factores que, en el actual marco institucional, determinan su adquisición, y su relevancia moral, concebida no sólo como un estatus que comporta la atribución de cualificados derechos políticos y sociales sino también como factor generador de vínculos, dependencias y solidaridades entre un grupo de individuos. Algunos cosmopolitas niegan cualquier valor ético a la ciudadanía subrayando el modo en el que, tal y como está configurado el actual orden mundial (el que el ius soli es el principal título de adquisición de la nacionalidad), un hecho completamente azaroso como es el lugar de nacimiento resulte determinante, mucho más que cualquier otro factor (el género, la edad, la condición social, etc.) del nivel de oportunidades y opciones de vida valiosas de la que disponen las personas. Se hablado, por ello, de un "citizenship premium", para los que nacen en el sitio "correcto", y una "citizenship penalty", para los que lo hacen en los países "erróneos" (Milanovic 2016: 13; en un sentido similar, Velasco 2016; Shachar 2009; Stevens 2009). Sin embargo, una cosa es que el actual statu quo atribuya un peso desmedido al lugar de nacimiento frente a otros factores como la residencia en la adquisición de la nacionalidad/ciudadanía y otra muy distinta que el vínculo 
entre el nacimiento y la ciudadanía sea, per se, injusto y, sobre todo, que la ciudadanía carezca de relevancia ética.

Cuando nos preguntamos por el posible fundamento ético de unos deberes de justicia distributiva hacia los compatriotas, estamos tratando de identificar si hay algún elemento adicional a la humanidad compartida que justifique deberles algo más y/o distinto que al resto de los seres humanos. No buscamos cualquier tipo de diferencia o singularidad, sino una que permita sostener que la elección entre atender ciertas necesidades no fundamentales de los compatriotas y las más básicas de las personas que no forman parte de nuestra comunidad política puede ser descrito también como un conflicto entre deberes éticos diferentes y, al menos en cierta medida, independientes. Sirviéndonos de la expresión utilizada por Caney, buscamos alguna "disanalogy property" (Caney 2005: 271).

Hace tiempo que los principales discursos dirigidos a justificar los deberes redistributivos entre conciudadanos -y una hipotética preferencia de tales responsabilidades frente a las de la justicia global- han dejado de atender a los vínculos basados en la identidad compartida o la existencia de un cierto grado de no extrańeza entre los conciudadanos para centrarse, exclusivamente, en el marco institucional en que estos interactúan y relacionan. Asumiendo una concepción relacional de la justicia distributiva, han profundizado en el modo en que las instituciones políticas ya existentes generan una necesidad de igualdad comparativa sui generis entre sus participantes. De acuerdo con esta visión, sería el tipo de relaciones y dependencias únicas que surgen entre individuos como resultado de interactuar y cooperar en el marco de las comunidades políticas estatales lo que convierte al ámbito de los compatriotas en una esfera diferente de la justicia (Miller 2010: 40). Un sistema social y político que permitiese un grado excesivo de desigualdad entre ellos resultaría inaceptable para los menos aventajados. ¿Por qué razón? Algunos creen que la respuesta debe buscarse en el carácter coactivo de las comunidades políticas estatales. Supuestamente, el hecho de tener una relación coactiva con los compatriotas nos situaría en una relación moral especial con un conjunto de personas que no compartimos con todos los individuos (Blake 2002).

A mi juicio, no sería la coacción sin más lo que convertiría a las desigualdades domésticas en algo moralmente problemático (Arcos 2017), sino las relaciones y dependencias sociales, económicas y políticas que surgen entre ellos en tanto que participantes en un esquema de cooperación configurado por el Estado. Aunque la cooperación no tiene lugar únicamente entre los individuos o grupos que viven dentro de una misma comunidad política sino que, cada vez más, es una realidad transfronteriza, lo cierto es que la que tiene lugar entre conciudadanos resulta especial por su mayor amplitud e intensidad. 
El cosmopolitismo que aboga por extender globalmente los principios de la justicia doméstica parece ignorar el hecho de que los bienes públicos que permiten a los individuos desarrollar vidas satisfactorias (derechos, orden, seguridad, prestaciones sociales, acceso a diversas redes de intercambio económico, social y cultural, etc.) no son un "maná caído del cielo", sino únicamente el producto de una cooperación políticamente organizada y protegida (Pevnick 2011: 13 y 116). Para que este sistema resulte justo, es necesario que quienes están dispuestos a cooperar reciban a cambio dos contrapartidas. La primera de ellas, sin duda la más obvia, que existan mecanismos para prevenir y reprimir la aparición de free riders o gorrones, dispuestos a beneficiarse de las ventajas del sistema sin asumir sus costes. En segundo lugar, para que el sistema de cooperación resulte aceptable, quienes ocupan un lugar menos aventajado dentro de éste han de contar con que sus conciudadanos les mostrarán una preocupación especial respecto al resto de los seres humanos. Ello responde, por un lado, a que son ellos y no los extranjeros quienes contribuyen a mantener dicho esquema y, por otro, a que la posición aventajada de la que disfrutan los primeros es, en gran medida, resultado de una prosperidad alimentada por los bienes y oportunidades proporcionados por éste y no única, ni principalmente, de los esfuerzos privados (Miller 2010: 47-48) ${ }^{2}$. De ahí, que hayan de contar con que los beneficios derivados de la cooperación no estén abiertos a todos, ya sea a través de trasferencias de dinero dirigidas a la ayuda al desarrollo o la apertura de las fronteras. De lo contrario, quedarían desprovistos de incentivos para continuar formando parte de dicho sistema (Pevnick 2011: 110; Miller 2007: 70).

Uno de los primeros desarrollos de esta filosofía lo encontramos en lo que Rawls y Anderson llaman una concepción democrática de la igualdad distributiva. Desde este punto de vista, la igualdad socioeconómica sería una exigencia de que la brecha entre ricos y pobres no exceda de lo permitido por el ideal de la reciprocidad democrática, evitando con ello la estigmatización social y el trato como inferiores de los segundos (Anderson 1999: 313; Rawls 2001:139). Tal visión justificaría, tanto que este valor opere únicamente en las comunidades políticas estatales y las relaciones entre conciudadanos, como - de modo más velado- otorgar cierta prioridad a estas últimas responsabilidades frente a los deberes generales respecto a todos los seres humanos (Freeman 2003: 50).

La noción de incentivos para los menos aventajados resulta, no obstante, muy vaga. Cabría interpretarla en un sentido puramente político y no moral, como

\footnotetext{
2 Asumiendo la crítica de Goodin (1998: 687), algunos defensores de este argumento son conscientes de que justificaría extender dicha preferencia no sólo a los conciudadanos sino también a quienes no siéndolo (residentes permanentes) participan en el mismo esquema de cooperación social (Sangiovanni 2007: 38).
} 
el precio a pagar por los más aventajados para evitar que los que estén peor "no se salgan" del sistema o traten de cambiarlo, incluso, por la fuerza. Esto nos retrotrae a los orígenes del Estado Social como producto del acuerdo entre el capitalismo y la clase trabajadora por el que ésta renunciaba definitivamente al ideal revolucionario a cambio de una mejora sustancial de sus condiciones de vida a través de las políticas públicas asistenciales universales. Para otros, la visión de la preferencia como estímulos o incentivos parece aproximar su fundamento moral a una concepción de la justicia basada como reciprocidad, en la línea propugnada por Gauthier. Sin embargo, en el caso de Rawls y Anderson, parece más razonable pensar que estrían abogando por una visión de la justicia como fair reciprocity (Buchanan 1990).

Por otra parte, un ingrediente fundamental de la legitimidad democrática de los esquemas cooperativos desarrollados en el marco de los Estados está relacionado con lo que se conoce como prerrequisitos de la democracia. Esta no se reduciría a un conjunto de mecanismos para agregar preferencias a través de la regla de la mayoría, o, más idealmente, para racionalizarlas y moralizarlas a través de la discusión y el diálogo (democracia deliberativa), sino que también se proyecta sobre las relaciones sociopolíticas de los ciudadanos y sobre las razones que hacen que la estructura básica de la sociedad resulte aceptable para sus miembros menos aventajados. Para que en un esquema de cooperación que proporciona bienes públicos como los señalados sea justo, debe asegurar el derecho a la participación política no sólo formal sino también efectiva de todos los participantes en el mismo. Por consiguiente, no basta con la igualdad política para lograr tal justificación. Es necesario, además, garantizar cierto grado de igualdad económica a través, entre otras medidas, de un cierto grado de preferencia de los compatriotas (Klosko 2009: 259).

\section{La ponderación entre la justicia doméstica y las responsabilidades globales}

Habiendo examinado en Qué sentido y sobre la base de qué fundamentos los deberes hacia los compatriotas podrían ser considerados razones morales independientes, podemos enfrentarnos ahora a la cuestión inicialmente planteada: si, en el caso de que entren en conflicto y hubiera que priorizar a una sobre otra, la justicia doméstica debería ser antepuesta a la global. En el escenario que plateamos, no sería posible incrementar, de acuerdo con lo exigido por una redistribución igualitaria, lo que debemos a los compatriotas sin perjudicar la ayuda que debemos a los extranjeros, no sería posible mejorar sin priorizar (Abizadeh 2016: 121). En tal contexto, asumida la independencia 
o autonomía de ambos tipos de razones éticas, considero que el conflicto no debería ser resuelto en abstracto, otorgando prioridad absoluta o lexicográfica a una u otra de acuerdo con el principio the-winner-takes-it-all (García Gibson 2016). Este sería el modelo que defienden algunos cosmopolitas que sostienen que los deberes globales siempre han de prevalecer sobre los domésticos (Tan 2004: cap. VII). Más difícil es encontrar este tipo de prioridad lexical a la inversa, entre los partidarios de otorgar preferencia a los compatriotas. Quizás las reflexiones de Richard Miller se aproximen a esta posición (Miller 1998; Miller 2010: 53-54). Frente al modelo de prioridad absoluta, considero que las tensiones entre los deberes domésticos y los globales deberían de ser afrontadas a través de una ponderación basada en la proporcionalidad, un método que no sería únicamente aplicable a los conflictos entre razones que reclaman idéntico peso moral sino, más modestamente, algún valor moral (Reiff 2009). $\mathrm{Y}$, aunque, como podrá observarse, la asignación de peso a los deberes domésticos y globales tome en consideración criterios deontológicos, creo que, último término, el factor decisivo a la hora de establecer alguna prioridad entre ambos sería el equilibrio entre los beneficios que se obtienen dando preferencia al cumplimiento de una exigencia y los perjuicios que se derivan de la no satisfacción de la otra. Partiendo de las aportaciones de teóricos que defienden conclusiones muy diferentes sobre este tema (Miller 1998: 465; Miller 2007: 45-46; Abizadeh y Gilabert 2008: 363), los criterios de ponderación podrían ser algunos de los siguientes:

1) Lo significativos que sean los vínculos entre A y B.

2) La seriedad de la situación de $\mathrm{C}$ y el grado de disparidad de recursos entre C, por un lado, y A y B, por otro.

3) Si la situación en cuestión impone a A deberes positivos respecto a C o también deberes negativos. Ello vendrá determinado, fundamentalmente, por la determinación de las causas de la situación de necesidad de C.

4) Si la situación en la que se encuentra $\mathrm{C}$ es atribuible a sus elecciones o a las circunstancias.

5) Si las responsabilidades distributivas globales de A son consideradas exigencias de justicia o de beneficencia. 
6) La magnitud de los beneficios que proporcionaría a $\mathrm{C}$ otorgar preferencia a los deberes globales y de los perjuicios que ello ocasionaría a B. Un factor determinante para su cálculo sería el número de personas beneficiadas y perjudicadas.

En relación con el primer criterio de ponderación, no hay duda de que las relaciones entre conciudadanos no poseen los rasgos de intimidad, afectividad o cercanía característicos de los vínculos entre familiares y allegados. Empero, he concluido que los compatriotas participan en esquemas de cooperación cuya legitimidad exige, por razones de reciprocidad, que las desigualdades sociales y económicas entre los participantes en el mismo deban ser corregidas de acuerdo con algún principio de igualdad comparativa. Aunque resulte evidente que no son el tipo de razones que haría triunfar inexorablemente a los deberes especiales frente a los generales, sí podría decidir en ciertos casos en los que, después de aplicar el resto de los criterios, compatriotas y extranjeros seguirían muy igualados.

\subsection{La justicia global reparativa}

COMO SE DESPRENDE DEL TERCER CRITERIO señalado, el peso de los deberes globales frente a la justicia socioeconómica doméstica sería mucho mayor, y conllevaría exigencias distributivas más ambiciosas si su fundamento fuese la violación previa de un deber negativo de no dañar. Este es el camino que, como es sabido, ha seguido Thomas Pogge para construir su teoría de la justicia global. Su principal tesis es que, al participar en la instauración de un orden mundial que es el principal causante de la pobreza severa que padecen millones de seres humanos, tanto los gobiernos como los ciudadanos del primer mundo venimos violando dicho deber negativo y lesionando los derechos humanos más básicos de otras personas (Pogge 2002: 33).

Supeditar el peso de los deberes distributivos globales a la violación de deberes negativos en el modo defendido por Pogge resulta, sin embargo, una estrategia discutible. Por un lado, ello presupone unas cadenas causales que no son fáciles de seguir y clarificar, en las que no es posible demostrar qué factor es, a la postre, el determinante de la situación en la que se encuentran los pobres a los que debemos ayuda. Ciertamente, el orden global contiene elementos perjudiciales para los más pobres, entre otros, permitir a los países ricos aprovecharse en su beneficio de la posición privilegiada que ocupan en la negociación de los principales acuerdos comerciales regionales y mundiales, o no impedir la proliferación de los paraísos fiscales, favoreciendo con ello los flujos financieros ilícitos procedentes de la corrupción imperante 
en los países más pobres (Pogge 2011: 29-30). Empero, de ello no puede concluirse, ni que el orden global daña a los pobres, ni que sea la principal causa de la pobreza mundial (Risse 2005: 9-10). Aunque con matices significativos, la impresión dominante es que estamos ante un problema resultante no sólo de las injusticias globales sino también de una mala gobernanza doméstica (Lomasky y Teson 2015: 14).

De ahí que la teoría defendida por Pogge no resulte satisfactoria por varias razones. En primer lugar, al situar su fundamento en la obligación de compensar los dańos que provocamos a los pobres de todo el mundo mediante nuestro apoyo al orden internacional, la privación absoluta sólo se convertiría en una cuestión que nos incumbe moralmente en la medida, y únicamente en la medida, en que podamos ser considerados sus causantes, ya sea directa o indirectamente, a través de las estructuras sociales de las que nos beneficiamos. Esto significaría que no tenemos otras razones para apoyar una justicia redistributiva global que las basadas en la reciprocidad (en términos de compensación del daño verdaderamente causado a los más pobres), sin ninguna consideración a la preocupación que debe merecernos el sufrimiento y la dignidad de todos y cada uno de los seres humanos, cualquiera que sea la causa de sus padecimientos y de la relación que hayamos tenido con esa causa y con las víctimas. En segundo lugar, si el principal factor determinante de las responsabilidades distributivas hacia los más pobres debiera buscarse en las causas de la pobreza, quienes defienden la preferencia de los compatriotas y el derecho soberano de los Estados a controlar las fronteras, podrían salir reforzados. Para estos no resultaría problemático admitir un principio similar -o incluso más ambicioso que el de Pogge- ya que, al defender una visión endógena de las causas de la pobreza global, no concurrirían las condiciones fácticas para que resulte operativo.

\subsection{Las responsabilidades distributivas globales ¿beneficencia o justicia?}

LA MAGNITUD DE LAS DESIGUALDADES de recursos y oportunidades entre quienes viven en distintos lugares del planeta ha de traducirse, inexorablemente, en la asignación de responsabilidades éticas de primer orden sobre quienes viven en condiciones de vida mucho mejores. Lo que se discute, pues, no es si tenemos deberes positivos respecto a los extranjeros, sino si el fundamento de estos últimos sería similar al de los de la justicia doméstica. Mientras los cosmopolitas más genuinos estiman que sería el mismo (la igualdad en sentido comparativo), para los moderados la justicia global adoptaría como criterio distributivo la eliminación de la privación absoluta y no de la relativa. Este es, en último término, el centro de los debates sobre la justicia global: si ésta deber igualitarista o suficientista (Tan 2014: 207). 
Algunos cosmopolitas estiman que el dualismo ético que suscriben los cosmopolitas moderados constituye, en sí mismo, una forma de prioridad de los compatriotas (Ypi 2013: 77-78). Para otros, se traduce en otra forma de entender este favoritismo: que el cumplimiento de los deberes distributivos respecto a los compatriotas tendría preferencia sobre las responsabilidades globales. Esta convicción estaría alimentada por la creencia en que la preocupación por la privación absoluta no es un deber de justicia sino de humanidad o beneficencia. Esta última caracterización resulta ser, aparentemente, decisiva de cara a determinar la fuerza de las responsabilidades hacia los extranjeros. Si, además de sostener que no se basan en la igualdad comparativa sino en principios distributivos como la suficiencia o la prioridad, también las consideramos como exigencias de humanidad, con un trasfondo ético como la compasión o la caridad y un cumplimiento no exigible sino solo opcional, su fuerza parecería ser inferior a la de los deberes de la justicia doméstica (Miller 2007: 248).

Las premisas en las que se apoya esta última justificación de la preferencia de los compatriotas no son, por diferentes razones, aceptables. En primer lugar, no parece razonable considerar a la suficiencia o la prioridad como principios distributivos de humanidad y no de justicia (Mason 2006: cap.V; Gilabert 2012: 4-5). Muchos piensan que ésta no adopta únicamente una forma igualitaria y comparativa, sino que también puede basarse en principios que exijan mostrar preferencia por los que están peor, con independencia de lo mucho o poco que les beneficie al hacerlo (prioridad), o mientras no se encuentren en condiciones de acceder a una vida decente (suficiencia). Por lo tanto, el conflicto entre las responsabilidades domésticas y las globales no se produciría entre deberes de justicia y de humanidad, sino entre distintos deberes justicia. En segundo lugar, conviene diferenciar tres dimensiones de los deberes éticos: su alcance (doméstico o global), su contenido (igualitario, suficiencista, prioritarista, etc.) y su peso o fuerza. Aun cuando consideremos a las responsabilidades globales como deberes de humanidad, no cabe concluir que sean exigencias con menos fuerza que, en caso de colisionar con los deberes igualitarios domésticos, resulten siempre derrotadas por estos (Campbell 1976:14; Loriaux 2006: 253).

Con independencia de que remover las causas de la privación absoluta, y no sólo relativa, también exija a corto o medio plazo reducir considerablemente la escandalosa disparidad de recursos existente a nivel global, el objetivo de satisfacer las necesidades más básicas de los más pobres del planeta -facilitándoles el acceso a bienes tan esenciales como agua limpia y potable, abrigo, cobijo, educación, atención médica, etc.- sería un objetivo de signo aparentemente suficientista que exige "más que la asistencia estatista, pero menos que la igualdad cosmopolita a gran escala" (Valentini 2011: 40). Si uno de los factores a tener en cuenta a la hora de 
ponderar deberes en conflicto es la gravedad o seriedad de su contenido, parece difícil sostener -ya sea sobre la base de principios utilitaristas basados en la reducción del sufrimiento, de la adopción de un punto de vista imparcial que otorgue un valor intrínseco e igualitario a las necesidades más básicas y urgentes de las personas, o de argumentos contractualitas centrados en la noción de derechos humanos- que los deberes globales sean menos intensos que los domésticos y no lo contrario.

\subsection{Costes y beneficios de anteponer la reducción de la pobreza global a la igualdad doméstica}

El ÚlTIMO CRITERIO DE PONDERACIÓN que tomaré en consideración es el relativo a la magnitud de los costes que impondría a los países ricos afrontar las responsabilidades globales (en particular, mediante la apertura de las fronteras) y la magnitud de los beneficios que ello reportaría a quienes padecen privación extrema (Barry y Wiens 2020). Respecto a lo último, me limitaré a constatar que todas las medidas redistributivas ideadas para acabar con ésta, o para reducir las desigualdades globales, tendrían, al menos prima facie, efectos beneficiosos para los más pobres, con independencia de cuál sea la forma en que se midan o calculen.

Más controvertido se presenta, por el contrario, el problema de los costes de la erradicación de la pobreza extrema. Quizás el problema sea más complejo y no sea tan evidente que acabar con la privación absoluta resulte sea tan fácil y poco costoso como sostiene algunos cosmopolitas. Si concedemos que ésta no se refiere únicamente a los ingresos, sino que es un fenómeno multidimensional, relacionado también con la salud, la educación, el alojamiento o la participación, entonces erradicarla podría resultar un objetivo algo más complejo. Sostendré, pues, que los costes, tanto de la apertura de las fronteras, como de cualquier otro medio para hacer efectivas las responsabilidades distributivas globales, no serían tan poco significativos como para negar que pudieran imponer ciertos sacrificios a los países más ricos.

Como comentamos al comienzo, algunos sostienen que la apertura de las fronteras pondría en peligro los programas asistenciales del Welfare State y empeoraría las condiciones salariales de los trabajadores domésticos. Los datos que arrojan los estudios sobre el impacto socioeconómico de la inmigración allí donde se han abierto significativamente las fronteras alimentan opiniones contradictorias, en particular en lo referente a los efectos que una llegada masiva de trabajadores no cualificados extranjeros tendría sobre el mercado de trabajo. Algunos estiman que la "wage competition" entre ciudadanos e inmigrantes ya ha provocado la bajada de los salarios de los trabajadores no cualificados (Borjas y Katz, 2007). Otros, por lo 
que se refiere especialmente a los Estados Unidos, señalan que dicho impacto sería muy modesto y afectaría a sectores muy concretos (Blau y Mackie 2015; Lomasky y Teson 2015; Fetzer 2016). Más polémica se presenta la cuestión de cómo afectaría un incremento muy significativo del número de inmigrantes a los servicios sociales. Aunque no hay evidencias de que estos hagan un uso mucho mayor de los mismos que el resto de la población (Brock 2009), no puede descartarse que una apertura de las fronteras, acompañada de unas prestaciones sociales generosas en educación, sanidad y, sobre todo, desempleo, tuviera un "welfare magnet" que haría bastante problemática la relación entre el Welfare State y la inmigración (Entzinger 2014). Se ha hablado, por ello, de una immigration/welfare paradoxe, para referirse al hecho de que la inmigración demanda fronteras más abiertas, mientras que el Estado social funciona mejor con fronteras cerradas.

No resulta fácil hacer un balance general de los costes y beneficios que conllevaría una apertura mucho mayor de las fronteras. Sus defensores podrían señalar que, aunque la llegada de más inmigrantes pudiera perturbar durante algún tiempo el funcionamiento del Welfare State, los efectos netos de la inmigración serían claramente beneficiosos para las sociedades de acogida: llegada de trabajadores cualificados a coste de formación cero, rejuvenecimiento demográfico, ocupación de puestos de trabajo no deseados por los nacionales del país, etc. (Brock 2009: 198). Sin embargo, puesto que los estudios existentes sólo han podido valorar las consecuencias de la apertura en ámbitos regionales limitados, no puede pronosticarse qué ocurriría si las fronteras fueran completa o significativamente abiertas para todos los habitantes del planeta. No puede descartarse que, sin llegar a los extremos apocalípticos que los más pesimistas han presagiado, al menos a corto plazo, la apertura pudiera tener costes importantes.

También la ayuda al desarrollo, el otro principal mecanismo para cumplir con las responsabilidades distributivas globales parece imponer sacrificios significativos a los países ricos. En la práctica, los gobiernos del primer mundo se escudan en la imposibilidad de hacer compatible el objetivo de una justicia social doméstica que garantice el acceso universal e igualitario a las prestaciones sociales básicas (sanidad, educación, seguridad social, etc.) y aumentar o incluso (en el marco de una crisis económica) mantener las partidas dedicadas a la ayuda al desarrollo. Desde finales de los ochenta, ha ido disminuyendo progresivamente el monto destinado a esta, hasta el punto de que en 2018 solo cinco de los países más aventajados cumplieron el compromiso alcanzado por la Amable general de la ONU en 1980 de invertir el 0,7\% de su PIB en cooperación al desarrollo: Dinamarca, Suecia, Noruega, Luxemburgo y Reino Unido, según los datos de la ODCE. La primera economía del mundo, Estados Unidos, sólo destinó el 0,17\%. 
Admitamos, en aras del argumento de la prioridad de los compatriotas, que -ya sea a través de la apertura de las fronteras, o del aumento de la ayuda exteriorel cumplimiento de las responsabilidades distributivas globales pudiera conllevar costes significativos para los estados más aventajados. ¿Serían dichos costes tan significativos como para imposibilitar satisfacer las necesidades básicas de sus ciudadanos? Evidentemente no. Si fuera este el caso, resultaría difícil cuestionar que uno de los límites que dichos costes no podrían traspasar sería situar a los conciudadanos por debajo del umbral de una mínima suficiencia. De acuerdo con este principio, estaría justificado anteponer la satisfacción de las necesidades básicas de los compatriotas a las de los que no lo son (Miller 2007: 48-49; Oberman 2016: 35-56). En este caso, y solo en este, creo que los vínculos asociativos serían un criterio determinante para otorgar mayor peso a los deberes domésticos que a los globales, una conclusión que sería trasladable al problema de la apertura de las fronteras (Sangiovanni 2007: 35).

En el extremo opuesto, no creo que pueda afirmarse de forma concluyente que los países ricos dispongan siempre de un "excedente de opulencia" (Van der Ven 2008: 422), que les permita, al mismo tiempo, mejorar ostensiblemente el nivel de cumplimiento de sus deberes globales y profundizar en la igualdad comparativa entre sus ciudadanos. Las demandas de justicia social poseen un carácter abierto, que impide señalar un límite evidente a los recursos que deberíamos destinar a la eliminación de las desigualdades en ámbitos como el acceso a la seguridad social, la salud, el mercado de trabajo o la educación (Rawls 1999: 106; Miller 2008: 562). Por tanto, parece difícil negar que puedan surgir conflictos entre la búsqueda de una mayor igualdad doméstica y la erradicación de la pobreza global. Si trasladáramos el problema al plano de la moral individual, un altruismo más que mínimo como el defendido por Singer (2016: 5-6), centrado en la capacidad de ayuda del agente para evitar algo malo sin sacrificar nada moralmente comparable, decantaría la balanza del lado de los más pobres. En el caso de una ética pensada para las instituciones, las cosas no parecen, sin embargo, tan fáciles. En los supuestos de privación absoluta más extrema (hambre, desnutrición severa, privación de asistencia sanitaria básica, etc.), en la que no pueden cubrirse las necesidades mínimas que hacen posible la supervivencia, parece difícil cuestionar la preferencia de las responsabilidades extra republicam. La seriedad de la situación en la que se encuentran los pobres globales, el número de personas que se encuentran muy mal, y la magnitud del beneficio que les reportaría la ayuda en comparación con la del sacrifico que ésta ocasionaría a los más ricos, exigiría otorgar más peso a los deberes globales que a los domésticos. Por lo que se refiere a las fronteras, se impondría, en estos casos, el reconocimiento de un derecho humano a inmigrar (Arcos 2020). 


\section{Consideraciones finales}

El DeSAFío QUE LA PRIORIDAD de los compatriotas representa para la justicia global y, en particular, para la apertura de las fronteras, se construye a partir de las siguientes premisas: a) no sólo los deberes distributivos globales sino también los que tienen como destinatarios a los conciudadanos constituyen razones morales independientes; b) aunque en la teoría, conceptualmente, los dos tipos de responsabilidades no sean incompatibles, en la práctica pueden surgir conflictos entre la observancia simultánea de ambas; c) los deberes patrióticos justificarían desatender las responsabilidades distributivas globales a través, entre otras medidas, de una restricción severa de la entrada de inmigrantes, si esta última conlleva perjuicios para los conciudadanos menos aventajados. A estos presupuestos normativos cabría ańadir una premisa empírica: d) la apertura de las fronteras, en especial a los trabajadores poco cualificados, tiene efectos perniciosos sobre los menos aventajados de las sociedades de acogida.

Diversas críticas al favoritismo de los compatriotas se han centrado en desacreditar la premisa empírica del argumento, sirviéndose de diversos estudios sociológicos y económicos que demostrarían que la llegada de inmigrantes no posee ninguno de los efectos negativos antes señalados. Frente a este planteamiento, he asumido que un aumento significativo de la entrada de inmigrantes podría tener costes significativos para los miembros menos aventajados de una comunidad política próspera. Por tanto, en contra de la habitual tendencia de los teóricos de la justicia global a ignorar este tipo de escenarios, estimo que la apertura de las fronteras sería uno de los supuestos en los que las lealtades patrióticas y las responsabilidades distributivas globales pueden colisionar.

Admitida la existencia de tales conflictos, he señalado las razones por las que las responsabilidades distributivas globales tendrían peso suficiente para imponerse en muchos contextos a las demandas de una mayor igualdad doméstica, así como para exigir a los países ricos actuaciones muy diversas con vistas a facilitar el acceso de los peor situados del planeta a unas condiciones de vida mínimamente decentes. Aunque ello pueda conllevar ciertos costes, no serían tan significativos en comparación con el beneficio que proporcionaría a los primeros, cualquiera que sea la "aritmética moral" con la que se mida. Por lo tanto, el argumento de la prioridad de los compatriotas no podría servir de justificación para rechazar la apertura de las fronteras, si ésta demuestra ser un instrumento adecuado para tal objetivo. Por eso mismo, si uno de los principales avales éticos de dicha medida reside en su función redistributiva de la riqueza global, cualquier intento de justificarla debería demostrar no sólo que sus costes no serían excesivos, sino además que no existe ninguna medida 
alternativa que permitiera aminorarlos y, sobre todo, realizar más eficientemente dicha función (Oberman, 2016: 50; Turégano 2019).

Si adoptamos una perspectiva radicalmente suficientista o prioritarista, o basada en un humanitarismo de emergencia, seguramente la forma más rápida de satisfacer las necesidades más básicas y urgentes de los más pobres exigiría dejarles entrar a formar parte del club y beneficiarse, como ciudadanos o residentes, de las oportunidades reservadas a los que ya están dentro. Sin embargo, ¿es la apertura de las fronteras el medio más efectivo para erradicar la pobreza extrema global a medio o largo plazo?

Dicha medida podría ser el medio más adecuado para mejorar la posición de los peor situados allí donde, entre los que están dentro y los que quieren entrar, existan interacciones e interdependencias sociales y económicas tan intensas y potencialmente opresivas, como para que su erradicación exija una distribución de las oportunidades y recursos basada en la igualdad comparativa. Tales consideraciones no serían, sin embargo, trasladables al resto de contextos globales en los que las relaciones e interdependencias no alcanzan dicha intensidad, y en los que la redistribución estaría, en principio, dirigida a satisfacer las necesidades más básicas de quienes sufren privación absoluta. Al contrario, hay razones para poner en duda que, en dichos contextos, las fronteras abiertas sean el medio más óptimo para descargar las responsabilidades distributivas globales y no solo un second-best. a) el coste de beneficiar a las personas a quienes su nivel de privación y falta de oportunidades les obliga a emigrar es mucho menor ayudándolas en sus sociedades de origen, donde el dinero trasferido posee mayor poder adquisitivo; b) para poder emigrar hace falta algo más que poner fin a las restricciones a la entrada de inmigrantes. Dado su nivel tan mínimo de ingresos (menos de $2 \$$ al día), la apertura de las fronteras no sería suficiente para que los más pobres pudieran de facto emigrar (Shachar 2009: 84-85). Esto explicaría, entre otras razones, que, en la en la práctica, levantar las restricciones a la entrada de inmigrantes no se traduzca únicamente en la llegada de trabajadores poco cualificados, sino, en mucha mayor proporción, de profesionales cualificados, con la consiguiente brain drain que tanto ha perjudicado a los países más pobres.

A la vista de estos hechos, las mismas razones que justifican anteponer la justicia global a la doméstica -los extranjeros que están peor y no pueden acceder a una vida mínimamente decente a los compatriotas menos aventajados que tienen satisfechas sus necesidades como mínimo básicas- termina convirtiéndose, paradójicamente, en el talón de Aquiles de la apertura de las fronteras. Por supuesto, esto no significa que esta medida, combinada adecuadamente con otras, no pueda jugar un papel significativo en los esfuerzos por erradicar la pobreza extrema, pero no parece deba ser la única ni la principal forma para tratar de alcanzar dicho objetivo. 


\section{Referencias Bibliográficas}

Abizadeh, Arash y Pablo Gilabert, 2008. Is there a genuine tension between cosmopolitan egalitarianism and special responsibilities?. Philosophical Studies 138: 349-365.

Abizadeh, Arash, Manish Pandey y Sohrab Abizadeh. 2015. Wage competition and the special-obligations challenge to more open borders. Politics, Philosophy \& Economics 14 (3): 255-269.

Abizadeh, Arash. 2016. The Special-Obligations Challenge to More Open Borders. En Migration in Political Theory, eds. L. Ypi y S. Fine, 105-124. Oxford: Oxford UP.

Anderson, Elisabeth. 1999. What is the Point of Equality?. Ethics 109 (2): 287337.

Arcos Ramírez, Federico. 2017. El desafío del igualitarismo doméstico a la apertura de las fronteras: el argumento de la singularidad de la coacción estatal. Cuadernos Electrónicos de Filosofía del Derecho 35: 1-18.

Arcos Ramírez, Federico. 2020. ¿Existe un derecho humano a inmigrar? Una crítica del argumento de la continuidad lógica. Doxa 43: 285-312.

Barry, Christian y David Wiens. 2020. What Second-Best Scenarios Reveal About Ideals of Global Justice. En Oxford Handbook to Global Justice, ed. T. Brooks, 10.1093/oxfordhb/9780198714354.013.16. Oxford: Oxford UP.

Bayón, Juan Carlos. 2014. Democracia más allá del Estado. En Entre el Estado y la Cosmópolis, ed. A. Ruiz Miguel, 121-138. Madrid: Trotta.

Beitz, Charles. 1999. Political Theory and International Relations. Princeton: Princeton UP..

Blake, Michael. 2002. Distributive justice, State Coercion, and Autonomy. Philosophy and Public Affairs 30 (3): 257-296.

Blau, Francine y Christopher Mackie. 2016. The Economic and Fiscal Consequences of Immigration. Washington: The National Academies Press.

Borjas, George y Lawrence Katz. 2007. The evolution of the Mexican-born workforce in the United State. En Mexican Immigration in the United States, ed. Georges Borjas, 15-36. Chicago: University of Chicago Press.

Brock, Gillian. 2009. Global Justice. Oxford: Oxford UP. 
Brock, Gillian. 2015. Global justice, Cosmopolitan Duties and Duties to Compatriots: The case of Healthcare. Public Health Ethics 8 (2): 110-120.

Buchanan, Allen. 1990. Justice as Reciprocity versus Subject-Centered Justice. Philosophy \& Public Affairs 19 (3): 227-252.

Cabrera, Luis. 2010. World government: Renewed debate, persistent challenges. European Journal of International Relations 16 (3): 511-530.

Campbell, Tom. 1976. Humanity before Justice. British Journal of Political Science 4 (1): 1-16.

Caney, Simon. 2005. Justice Beyond Borders. A Global Political Theory. Oxford: Oxford UP.

Chatterjee, Deen. 2011. Reciprocity, Closed-Impartiality, and National Borders. Social Philosophy Today 27: 199-215.

Couture, Jaqueline y Kai Nielsen. 2006. Cosmopolitanism and the compatriot priority principle. En The Philosophy of Cosmopolitanism, eds. H. Brighouse y G. Brock, 180-195. Cambridge: Cambridge UP.

Entzinger, Hans. 2007. Open borders and the Welfare State. En Migration Without Borders, eds. A. Pécoud y P. De Guchteneire, , 119-134. Nueva York: Bergham Books.

Fetzer, Joel. 2016. Open Borders and International Migration Policy. Basingstoke: Palgrave Macmillan.

Freeman, Samuel. 2003. Introduction: John Rawls - An Overview. En The Cambridge Companion to Rawls, ed. S. Freeman (ed.), 1-61. Cambridge: Cambridge UP.

Garcia Gibson, Francisco. 2016. Conflicts between domestic inequality and global poverty: lexicality vs. proportionality. Ethics \& Global Politics 9 (1): https:// doi.org/10.3402/egp.v9.29803.

Gilabert, Pablo. 2012. From global poverty to Global equality. Oxford: Oxford UP.

Goodin, Robert. 1998. What is So Special About Our Fellow Countrymen?. Ethics 98: 633-686.

Kagan, Shelley. 1991. The Limits of Morality. Oxford: Clarendon Press.

Klosko, George. 2009. Cosmopolitanism, Political Obligation, and the Welfare State. Political Theory 37 (2): 243-265. 
Kukathas, Chandran. 2014. The Case for Open Immigration. En Contemporary Debates in Applied Ethics, eds. A. Cohen y C. Wellman, 376-388. Chichester: Willey-Blackwell.

Lenard, Patti Tamara y Margaret Moore. 2009. Ineliminable Tension. Philosophical Studies 138: 349-65.

Lomasky, Loren y Fernando Teson. 2015. Justice at distance. Cambridge: Cambridge UP.

Loriaux, Sylvie. 2006. Beneficence and Distributive Justice in a Globalized World. Global Society 20 (3): 251-266.

Marchetti, Raffaele. 2008. Global Democracy. Londres: Routledge.

Mason, Andrew. 2006. Levelling the Playing Field. Oxford: Oxford UP.

Milanovic, Branko. 2016. Global Inequality. Cambridge, MA: The Belknap Press.

Miller, David. 2007. National Responsibility and Global Justice. Oxford: Oxford UP.

Miller, David. 2008. A Response. Critical Review of Social and Political Philosophy 11(4): 553-567.

Miller, Richard. 1998. Cosmopolitan respect and the Patriotic Concern. Philosophy and Public Affairs 27 (3): 202-224.

Miller, Richard. 2010. Globalizing Justice. Oxford: Oxford UP.

Nili, Shmuel. 2015. Who's afraid of a world state?. Critical Review of International Social and Political Philosophy 18 (3): 241-263.

Oberman, Kiera. 2016. Immigration as a human right. En Migration in Political Theory, eds. L. Ypi y S. Fine, 32-56. Oxford: Oxford UP.

Pevnick, Ryan. 2011. Immigration and the Constrains of Justice. Cambridge: Cambridge UP.

Pogge, Thomas. 2002. World Poverty and Human Rights. Cambridge: Polity Press.

Pogge, Thomas. 2011. Are We Violating the Human Rights of the World's Poor?". Yale Human Rights \& Development Law Journal 14 (2): 1-33.

Rawls, John. 1999. The Law of Peoples with "The Idea of Public Reason Revisited". Cambridge, MA: Harvard UP. 
Rawls, John. 2001. Justice as fairness. Cambridge, MA: Harvard UP.

Reiff, Mark. 2009. Proportionality, winner-take-all and distributive justice. Politics, philosophy \& economics 8 (1): 5-42.

Risse, Mathias. 2005. Do We Owe the Global Poor Assistance or Rectification? Ethics \& International Affairs 19 (1): 9-18.

Sangiovanni, Andrea. 2007. Global Justice, Reciprocity and the State. Philosophy \& Public Affairs 35 (1): 3-39.

Scheffler, Samuel. 2001. Boundaries and Allegiances. Oxford: Oxford UP.

Scheuerman, William. 2013. Cosmopolitanism and the World State. Review of International Studies, 1-23.

Seglow, Jontathan. 2010. Associative Duties and Global Justice. Journal of Moral Philosophy, 7: 54-73.

Seleme, Hugo. 2007. El peso del deber de asistencia a pueblos foráneos frente a las exigencias de justicia doméstica. Diánoia 52 (59): 97-126.

Sen, Amartya. 2009. The Idea of Justice. Londres: Penguin.

Shachar, Ayelet. 2009. The Birthright Lottery. Cambridge, MA: Harvard UP.

Shklar, Judith. 2018. El liberalismo del miedo. Barcelona: Herder.

Singer, Peter. 2016. Famine, Affuence and Morality. Oxford: Oxford UP.

Tan, Kok-Chor. 2004. Justice without borders. Cambridge: Cambridge UP.

Tan, Kok-Chor. 2013. Cosmopolitanism and Patriotism. En Rooted Cosmopolitanism, eds. W. Kymlicka y K. Walker, 31-46. Vancouver: UBC Press.

Tan, Kok-Chor. 2014. Sufficiency, Equality and the Consequences of Global Coercion. Law, Ethics and Philosophy 8: 190-209.

Turégano, Isabel. 2019. Ethical Dimensions of Migration Policies. En Challenging the Borders of Justice in the Age of Migrations, eds. J.C. Velasco y MC. La Barbera, MC., 95-116, Cham: Springer.

Valentini, Laura. 2011. Justice in a Globalized World. Oxford: Oxford UP.

Van der Ven, Robert. 2008. Reasonable partiality for compatriots and the global responsibility gap. Critical Review of Social and Political Philosophy 11 (4): 413-432.

Velasco, Juan Carlos. 2016. El azar de las fronteras. México: FCE. 
Wellmann, Cristopher. 2000. Relational Facts in Liberal Political Theory. Ethics 110: 537-562.

Wendt, Alexander. 2003. Why a World State is Inevitable? European Journal of International Relations 9 (4): 491-542.

Ypi, Lea, 2013. Cosmopolitanism Without If and Without But. En Cosmopolitanism versus Non-Cosmopolitanism, ed. G. Brock, 75-91. Oxford: Oxford UP.

DOI: http://dx.doi.org/10.15366/bp.2020.23.003

Bajo Palabra. II Época. No23. Pgs: 73-100 